\title{
Kidney Disease: Improving Global Outcomes (KDIGO) New Guideline for Diabetes Management in Chronic Kidney Disease: A Meet-the-Expert Session
}

\author{
This symposium took place on $22^{\text {nd }}$ September 2020 , as part of \\ the Virtual European Association for the Study of Diabetes \\ (EASD) Annual Meeting 2020
}

\author{
Chairperson: \\ Ian de Boer \\ Speakers: \\ Peter Rossing, ${ }^{2,3}$ Tami Sadusky \\ 1. Kidney Research Institute, Seattle, Washington, USA \\ 2. Steno Diabetes Center Copenhagen, Copenhagen, Denmark \\ 3. Department of Clinical Medicine, University of Copenhagen, Copenhagen, Denmark \\ Disclosure: \\ Prof de Boer has received consulting fees from Boehringer Ingelheim, George Clinical, \\ Goldfinch Bio, and Ironwood Pharmaceuticals; and research equipment and supplies \\ from Medtronic and Abbott. Prof Rossing has received consultancy and/or speaking \\ fees (to his institution) from Astellas Pharma, AstraZeneca, Bayer, Boehringer \\ Ingelheim, Eli Lilly and Company, Gilead, MSD, Novo Nordisk, Sanofi Aventis, and \\ Vifor; and has received research grants from AstraZeneca and Novo Nordisk. Ms \\ Sadusky has declared no conflicts of interest.
}

Acknowledgements: Writing assistance was provided by Stefan Amisten, Amisten Consulting Limited, Epsom, UK.

Support:

The symposium and publication of this article were funded by Boehringer Ingelheim and Eli Lilly and Company.

Citation:

EMJ Diabet. 2020;DOI/10.33590/emjdiabet/201014

\section{Meeting Summary}

Prof de Boer opened this virtual seminar on the new Kidney Disease: Improving Global Outcomes (KDIGO) clinical practice guideline on diabetes management in chronic kidney disease (CKD), which is the first set of KDIGO guidance on this topic. Prof de Boer emphasised that the aim of the guideline was to generate a useful resource for clinicians and patients, to address relevant questions with actionable recommendations supplemented by practice points, to take on controversial topics when sufficient evidence was available, and to communicate findings clearly and concisely. The scope of the new guideline includes patients with Type 1 diabetes mellitus, Type 2 diabetes mellitus, and all severities of CKD, including patients treated with dialysis or kidney transplantation. The new guideline also includes recommendations related to lifestyle, pharmacotherapy, and the organisation of healthcare systems, addressed using systematically identified data from randomised controlled trials. Topics such as blood pressure control and lipid management and prevention of and screening for diabetes are not covered by the new KDIGO guideline and have been addressed either in prior KDIGO publications or in other international guidelines.

After his introduction, Prof de Boer handed over to Prof Rossing, who offered a detailed overview of the new guidelines, and Ms Sadusky, who highlighted the contribution of patients in the 
development of the guidelines. Prof Rossing and Ms Sadusky concluded the seminar by emphasising the importance of shared decision-making, where the patient is involved in defining individualised treatment goals, and the critical need for a team-based approach in the care of patients with diabetes and CKD.

\section{Management of and Living with Diabetes and Chronic Kidney Disease}

\section{Professor Peter Rossing and Miss Tami Sadusky}

Diabetes is a chronic, metabolic disease characterised by elevated blood glucose levels, which over time leads to serious damage to the heart, blood vessels, eyes, kidneys, and nerves. The most common form of diabetes are Type 2 diabetes mellitus, characterised by insulin resistance or insufficient production of insulin; followed by Type 1 diabetes mellitus, which is caused by the loss of endogenous insulin production. ${ }^{1}$ CKD develops in approximately $40 \%$ of patients with diabetes and is the leading cause of CKD worldwide. ${ }^{2}$

KDIGO is a global nonprofit organisation that develops and implements evidence-based clinical practice guidelines for kidney disease. ${ }^{3}$ The first KDIGO guideline on diabetes management in CKD has recently been developed and its guidance and recommendations on comprehensive care in patients with diabetes and CKD includes glycaemic monitoring and targets, lifestyle, and pharmacologic interventions. ${ }^{4}$ It emphasises that patients should be treated with a comprehensive strategy beyond lowering glycaemia to reduce risks of kidney disease progression and cardiovascular disease by highlighting the importance of screening for and management of the complications of diabetes.

\section{The Importance of Patient Perspectives for Treatment Guidelines}

A new evolution in the development of the KDIGO guidelines is the explicit inclusion of patient perspectives. This new concept was implemented by the involvement of patients as members of the KDIGO Work Group. Ms Sadusky, community and patient advocate and KDIGO Diabetes Guideline Work Group Member, demonstrated the value of including a patient in the guideline development process by sharing her perspective on patient care, with an emphasis on the importance of early testing and diagnosis of diabetes and its complications, such as CKD. She also highlighted the importance of including the patient in the decision-making process for their care (i.e., shared decision-making between patient and healthcare provider) and ensuring that a healthy lifestyle (e.g., diet and exercise), individualised to each patient, is a priority in the care of patients with diabetes and CKD. Ms Sadusky also stressed that adding lifestyle considerations to the decision-making process reduces the risk of future complications, and that there is a critical need for a team-based approach by healthcare providers in the care of patients with diabetes and CKD.

\section{Glucose Monitoring}

Monitoring of glycaemic status by either the patients or healthcare providers helps to guide treatments to achieve the best possible glycaemic control. ${ }^{5}$ Long-term glycaemia may be monitored through HbA1c, glycated albumin, fructosamine, or 1,5-anhydroglucitol, ${ }^{6}$ but it has not been clear which method is best suited for monitoring long-term glycaemia in patients with diabetes and CKD. In the new guideline, KDIGO recommends HbA1c to monitor glycaemic control, as HbA1c is a long-term glycaemic marker with a thoroughly studied performance as both a marker and a prognostic indicator, with standardised assays being readily available. ${ }^{4}$

Daily glycaemic monitoring with continuous glucose monitoring or self-monitoring of blood glucose may help to prevent hypoglycaemia and improve glycaemic control when antihyperglycaemic therapies associated with risk of hypoglycaemia, such as insulin, sulfonylureas, and meglitinides, are used. ${ }^{4}$ Prof Rossing also pointed out that monitoring for hypoglycaemia is less necessary for antihyperglycaemics such as metformin, sodiumglucose co-transporter-2 (SGLT-2) inhibitors, dipeptidyl peptidase-4 inhibitors, and glucagonlike peptide 1 receptor agonists (GLP1RA). 


\section{Individual Treatment Targets}

The new KDIGO guideline emphasises balancing risks and benefits for personalised treatment goals and recommends an individualised $\mathrm{HbAlc}$ target ranging from $<6.5 \%$ to $<8.0 \%$ in patients with diabetes and CKD not receiving dialysis. The individualised HbAlc target most suitable for each individual patient depends on a variety of factors, including CKD severity, macrovascular complications, comorbidities, life expectancy, hypoglycaemia awareness, resources for hypoglycaemia management, and the propensity of treatments to cause hypoglycaemia (Figure 1). ${ }^{4}$ Despite controversy about the goals for 'many' or 'most' patients, there is general agreement that glycaemic targets should be individualised based on consideration of specific factors (Figure 2). More stringent control may be recommended if it can be achieved safely and with an acceptable burden of therapy and if life expectancy is sufficient to reap benefits of tight control, whereas less stringent control may be recommended if the life expectancy of the patient is such that the benefits of an intensive goal may not be realised, or if the risks and burdens outweigh the potential benefits. ${ }^{4}$

\section{Lifestyle Interventions}

The new KDIGO guideline recommends that patients with diabetes and CKD should consume an individualised diet high in vegetables, fruits, whole grains, fibre, legumes, plant-based proteins, unsaturated fats, and nuts, but lower in processed meats, refined carbohydrates, and sweetened beverages. KDIGO advocates a plate model, which can easily be adapted to different cultural contexts by substituting the visualisation of a plate for that of a rice bowl, injera, tortilla, or banana leaf. Regardless of the shape of the plate or its equivalent, the proportion of major food groups remains the same: $50 \%$ fruit and vegetables, $25 \%$ plant or animal protein, and $25 \%$ whole grain or starchy vegetables. ${ }^{4}$ The new KDIGO guideline suggests that sodium intake be limited to $<2 \mathrm{~g}$ per day (or $<5 \mathrm{~g}$ sodium chloride), consistent with the KDIGO guideline on blood pressure management and international guidelines on the prevention and treatment of CVD. ${ }^{7,8}$

The new guideline also recommends that patients with diabetes and CKD should be advised to undertake moderate-intensity physical activity for a cumulative duration of at least 150 minutes per week, or to a level compatible with their cardiovascular and physical tolerance. Patients should be advised to avoid sedentary behaviour, and physicians should consider advising and encouraging patients with obesity, diabetes, and CKD to lose weight, particularly patients with an estimated glomerular filtration rate $(e G F R) \geq 30 \mathrm{~mL} / \mathrm{min} / 1.73 \mathrm{~m}^{2} .{ }^{4}$

\section{Pharmacologic Treatment for Glycaemic Control and Kidney Protection}

After lifestyle intervention therapy centred on increased physical activity, improved nutrition, and weight loss, first-line therapy with metformin and a SGLT-2 inhibitor is recommended for patients with diabetes and CKD, and those with an eGFR $\geq 30 \mathrm{~mL} / \mathrm{min} / 1.73 \mathrm{~m}^{2}$. This guidance is based on findings reported from the recent CREDENCE study. ${ }^{9}$

For metformin, a reduced dose is recommended for eGFR $<45 \mathrm{~mL} / \mathrm{min} / 1.73 \mathrm{~m}^{2}$, with discontinuation at eGFR $<30 \mathrm{~mL} / \mathrm{min} / 1.73 \mathrm{~m}^{2}$. For patients with eGFR $\geq 45 \mathrm{~mL} / \mathrm{min} / 1.73 \mathrm{~m}^{2}$, both immediaterelease and extended-release formulations of metformin are recommended, with extendedrelease metformin recommended for patients experiencing gastrointestinal side effects by the immediate-release formulation. Dose uptitration over 7 days until the maximum dose has been reached is recommended for both formulations. Monitoring of vitamin B12 deficiency is recommended for patients who are either at risk of deficiency, or for patients who have been taking metformin for $>4$ years, regardless of kidney function. Annual kidney function monitoring is recommended for patients with eGFR $\geq 60 \mathrm{~mL} /$ $\mathrm{min} / 1.73 \mathrm{~m}^{2}$, whereas kidney function monitoring is recommended at least every 3-6 months for patients with eGFR $30-59 \mathrm{~mL} / \mathrm{min} / 1.73 \mathrm{~m}^{2}$. Dose adjustments may also be required for patients with eGFR $30-44 \mathrm{~mL} / \mathrm{min} / 1.73 \mathrm{~m}^{2}$. KDIGO also recommends that SGLT-2 inhibitor therapy should not be initiated in patients with eGFR $<30 \mathrm{~mL} /$ $\mathrm{min} / 1.73 \mathrm{~m}^{2}$, and that SGLT-2 inhibitor therapy should be discontinued in patients starting dialysis (Figure 3). ${ }^{4}$ For patients with eGFR $<45$ $\mathrm{mL} / \mathrm{min} / 1.73 \mathrm{~m}^{2}$, the primary treatment goal is not lowering glycaemia per se, but cardiovascular and kidney protection. 


\begin{tabular}{lcr}
\hline CKD G1 & Severity of CKD & CKD G5 \\
\hline Absent/minor & Macrovascular complications & Present/severe \\
\hline Few & Comorbidities & Many \\
Long & Life expectancy & Short \\
\hline Present & Hypoglycaemia awareness & Impaired \\
Available & Resources for hypoglycaemia management & Scarce \\
Low & Propensity of treatment to cause hypoglycaemia & Haigh
\end{tabular}

Figure 1: Factors influencing individualised HbA1c targets for patients with diabetes and chronic kidney disease.

CKD: chronic kidney disease; G: glomerular filtration rate category.

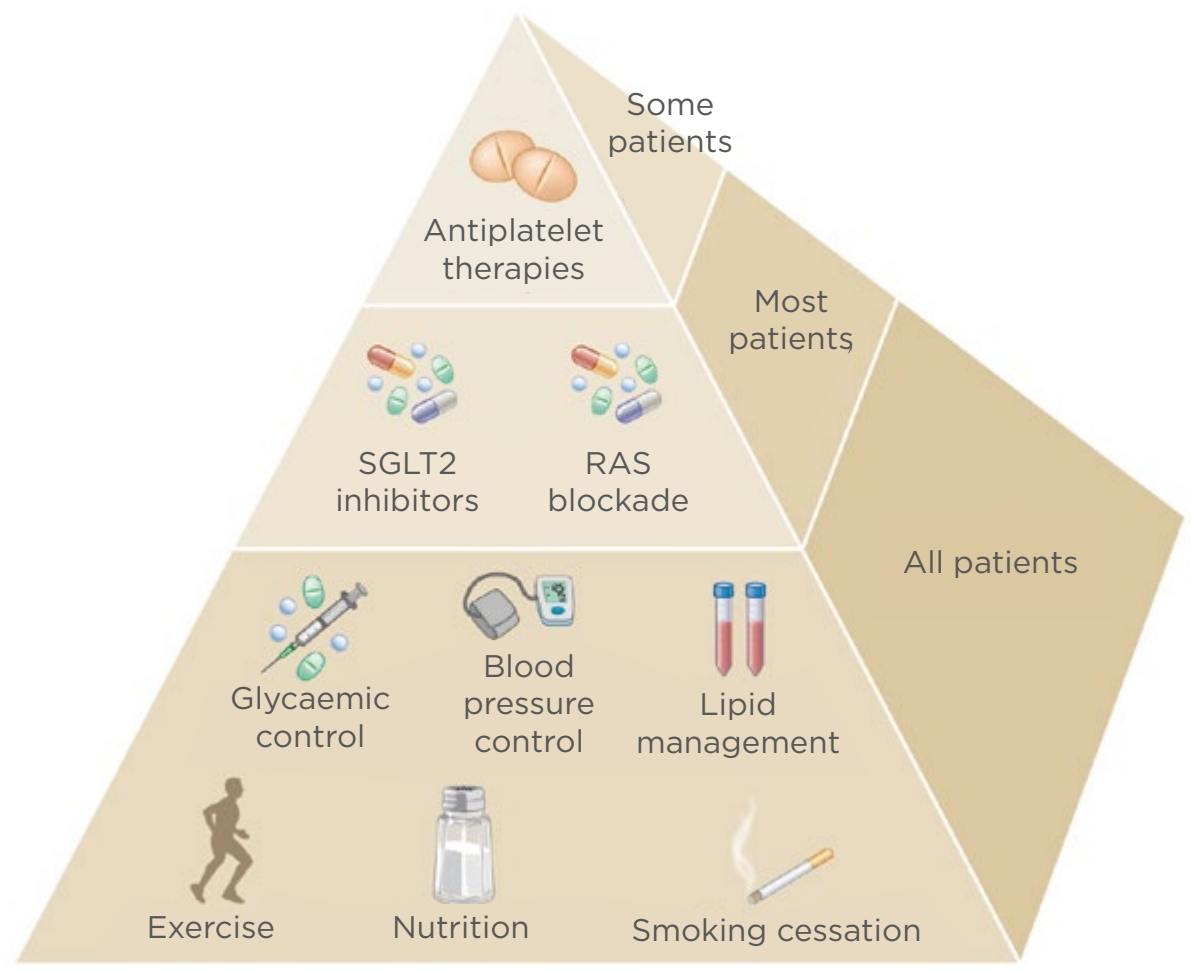

Diabetes with chronic kidney disease

Figure 2: Specific factors influencing individualised glycaemic targets for patients with diabetes and chronic kidney disease.

RAS: renin-angiotensin system; SGLT-2: sodium-glucose co-transporter-2.

Additional glucose-lowering therapies should be selected as needed for glycaemic control. These include GLP1RA (preferred), dipeptidyl peptidase-4 inhibitors, insulin, sulfonylureas, thiazolidinediones, or alpha-glucosidase inhibitors (Figure 3). Considerations for adding these therapies include patient preferences, comorbidities such as heart failure, high-risk atherosclerotic cardiovascular disease risk markers, need for potent glucose-lowering or reduced risk of hypoglycaemia, eGFR, cost, and other factors such as avoidance of injections, weight loss, renin-angiotensin system inhibitors (RAASi), and antiplatelet therapy. 


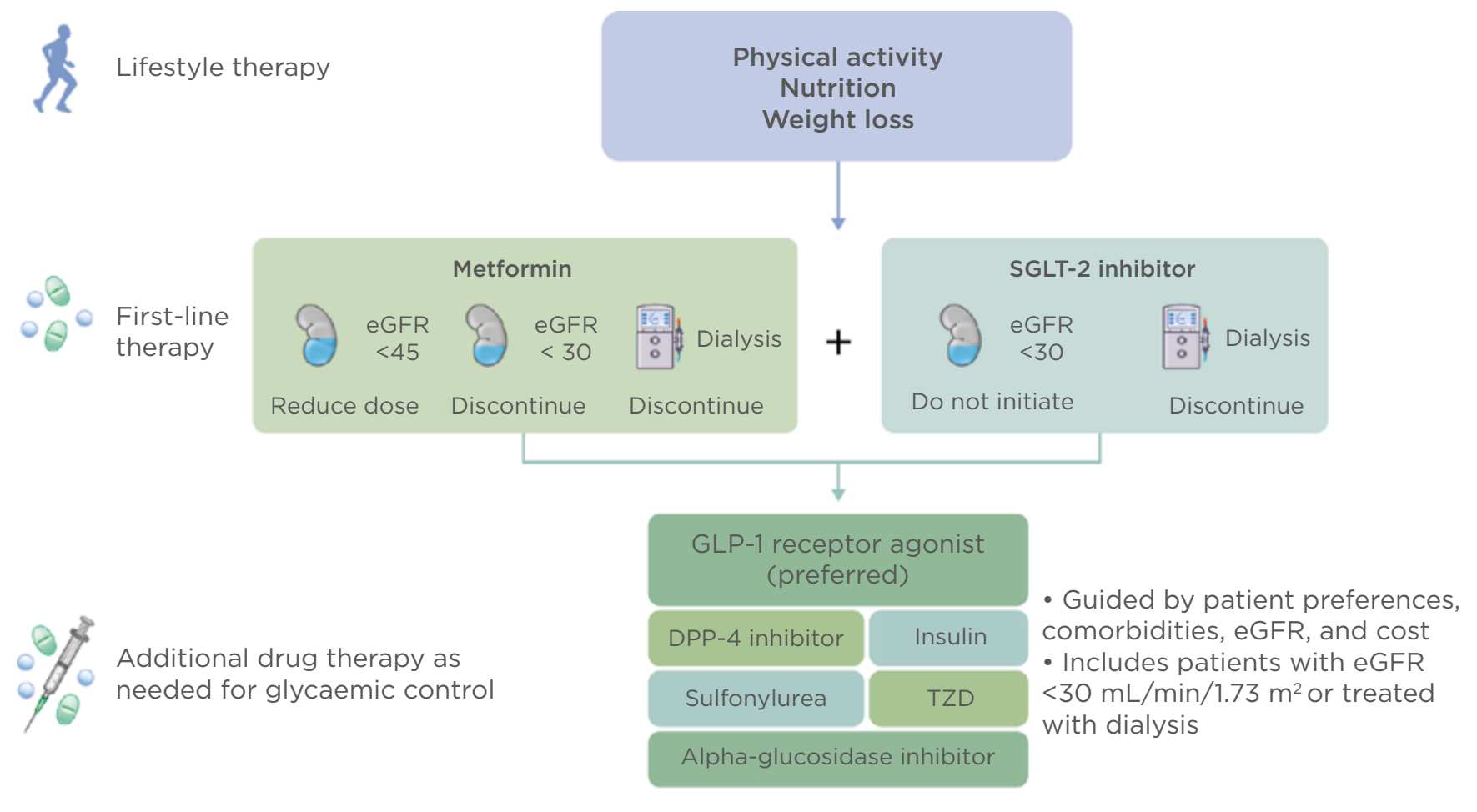

Figure 3: Overview of therapy options for patients with diabetes and chronic kidney disease.

eGFR: estimated glomerular filtration rate; SGLT-2: sodium-glucose co-transporter-2; GLP-1: glucagon-like peptide 1; DPP-4: dipeptidyl peptidase-4; TZD: thiazolidinediones.

\section{Patient Education}

As a preferred approach to the management of patients with diabetes and CKD, KDIGO recommends that a structured self-management educational programme be implemented for the care of individuals with diabetes and CKD.

The key objectives of such programmes are to improve diabetes-related knowledge, beliefs, and skills; improve self-management and self-motivation; encourage adoption and maintenance of healthy lifestyles; improve vascular risk factors; increase engagement with medication, glucose-monitoring, and complication screening programmes; reduce risk to prevent (or better manage) diabetesrelated complications; and to improve emotional wellbeing, treatment satisfaction, and quality of life. ${ }^{4}$

\section{The Importance of a Team-Based Approach}

The new KDIGO guideline also recommends that patients with diabetes and CKD should be treated using an integrated care approach to improve outcomes, self-management, and patientprovider communication. This approach consists of physician and other allied health professional care, supported by information technology to promote communication and feedback between specialists and other care providers. ${ }^{4}$ The aim of this care model is to achieve structured patient education and empowerment by improving selfmanagement and providing regular feedback to engage both patients and physicians.

In order to achieve this, KDIGO suggests that policymakers and institutional decision-makers should implement team-based, integrated care focussed on risk evaluation and patient empowerment to provide comprehensive care in patients with diabetes and CKD. ${ }^{4}$ The overarching goals are multi-target treatment for patients with diabetes and CKD (e.g., glycaemia, blood pressure, lipids), use of organ-protective drugs (e.g., RAASi, SGLT-2 inhibitors, GLP1RA, statins), and ongoing patient support to promote self-care. ${ }^{4}$ 


\section{Summary}

In summary, the new KDIGO guideline on the treatment of patients with diabetes and CKD provides recommendations and practice points on comprehensive care, with an emphasis on shared decision-making, in which the patient is involved in defining treatment goals best suited for them. It also provides guidance on lifestyle and antihyperglycaemic therapies for glycaemic control, the use of therapies such as SGLT-2 inhibitors to reduce the risk of cardiovascular and kidney complications, and the critical need for a team-based approach for the care of patients with Type 1 diabetes mellitus, Type 2 diabetes mellitus, and all severities of CKD, including patients treated with dialysis or kidney transplantation.

\section{References}

1. World Health Organization (WHO). Diabetes. Available at: https:// www.who.int/health-topics/ diabetes\#tab=tab_1. Last accessed: 8 October 2020

2. Alicic RZ et al. Diabetic kidney disease: challenges, progress, and possibilities. Clin J Am Soc Nephrol. 2017;12(12):2032-45

3. Kidney Disease: Improving Global Outcomes (KDIGO). 2020. Available at: https://kdigo.org/. Last accessed: 10 September 2020.
4. Kidney Disease: Improving Global Outcomes (KDIGO). Clinical practice guideline on the diabetes management in chronic kidney disease. 2020. [In press].

5. Goldstien DE et al. Tests of glycemia in diabetes. Diabetes Care. 2003;26(Suppl 1):s106-8.

6. Koga M. 1,5-Anhydroglucitol and glycated albumin in glycemia. Adv Clin Chem. 2014;64:269-301.

7. Arnett DK et al. 2019 ACC/AHA Guideline on the Primary Prevention of Cardiovascular Disease: Part 1 Lifestyle and Behavioral Factors. JAMA Cardiol. 2019;4(10):1043-4.

8. National Academies of Sciences, Engineering, and Medicine, Stallings VA, Harrison M, Oria M (eds.), Dietary Reference Intakes for Sodium and Potassium (2019), Washington, D.C. The National Academies Press.

9. Perkovic $\vee$ et al. Canagliflozin and renal outcomes in Type 2 diabetes and nephropathy. N Engl J Med. 2019;380(24):2295-306. 